\title{
Interpolating Sequences on the Bidisk
}

\author{
Jim Agler * \\ U.C. San Diego \\ La Jolla, California 92093 \\ John E. McCarthy \\ Washington University \\ St. Louis, Missouri 63130
}

\section{Introduction}

By an interpolating sequence for $H^{\infty}(\mathbb{D})$, the algebra of bounded analytic functions on the unit disk $\mathbb{D}$, we mean a sequence $\left\{\lambda_{i}\right\}_{i=1}^{\infty}$ in $\mathbb{D}$ with the property that, for any bounded sequence of complex numbers $\left\{w_{i}\right\}_{i=1}^{\infty}$, there is a function $f$ in $H^{\infty}(\mathbb{D})$ such that $f\left(\lambda_{i}\right)=w_{i}$ for each $i$. These sequences were characterized by L. Carleson in 1958 [10] (Theorem 0.1 below). To state Carleson's theorem, we need a few definitions.

Definition: The sequence $\left\{\lambda_{i}\right\}_{i=1}^{\infty}$ is weakly separated if there exists a constant $M$ such that, whenever $i \neq j$, there exists a function $\phi$ in $H^{\infty}(\mathbb{D})$ of norm at most $M$ that satisfies $\phi\left(\lambda_{i}\right)=1$ and $\phi\left(\lambda_{j}\right)=0$. The sequence is strongly separated if, for each $i$ there is a $\phi$ in $H^{\infty}(\mathbb{D})$ of norm at most $M$ that satisfies $\phi\left(\lambda_{i}\right)=1$ and $\phi\left(\lambda_{j}\right)=0$ for all $j \neq i$.

When dealing with $H^{\infty}(\mathbb{D})$, it is natural to consider it as the multiplier algebra of the Hardy space $H^{2}$, the Hilbert space of holomorphic functions on the disk with norm

$$
\|f\|_{H^{2}}^{2}:=\frac{1}{2 \pi} \lim _{r \nearrow 1} \int\left|f\left(r e^{i \theta}\right)\right|^{2} d \theta .
$$

The reproducing kernel for $H^{2}$ is the Szegő kernel

$$
s(\lambda, z)=\frac{1}{1-\bar{\lambda} z} .
$$

With respect to this kernel, the associated Gram matrix of the sequence $\left\{\lambda_{i}\right\}_{i=1}^{\infty}$ is the Grammian of the normalized kernel functions, i.e. the matrix with $(i, j)$ entry given by

$$
G_{i j}=\frac{s\left(\lambda_{i}, \lambda_{j}\right)}{\sqrt{s\left(\lambda_{i}, \lambda_{i}\right) s\left(\lambda_{j}, \lambda_{j}\right)}} .
$$

We can now state Carleson's theorem [10].

\footnotetext{
${ }^{*}$ Partially supported by the National Science Foundation
} 
Theorem 0.1 [Carleson] Let $\left\{\lambda_{i}\right\}_{i=1}^{\infty}$ be a sequence in $\mathbb{D}$. Then the following are equivalent:

(1) $\left\{\lambda_{i}\right\}_{i=1}^{\infty}$ is an interpolating sequence for $H^{\infty}(\mathbb{D})$.

(2) The sequence is weakly separated and the associated Gram matrix is bounded.

(3) The sequence is strongly separated.

Condition (2) is normally written differently. Rather than saying the associated Gram matrix is bounded, one says instead that the measure $\sum\left(1-\left|\lambda_{i}\right|^{2}\right) \delta_{\lambda_{i}}$ is a Carleson measure, i.e. there exists some constant $C$ such that, for all $f$ in $H^{2}$, the inequality

$$
\sum_{i=1}^{\infty}\left(1-\left|\lambda_{i}\right|^{2}\right)\left|f\left(\lambda_{i}\right)\right|^{2} \leq C^{2}\|f\|_{H^{2}}^{2}
$$

holds. The fact that boundedness of the Gram matrix is equivalent to a particular atomic measure being Carleson is true in great generality, and is proved in Proposition 1.1 below.

In 1987, B. Berndtsson, S.-Y. Chang and K.-C. Lin studied interpolating sequences for $H^{\infty}\left(\mathbb{D}^{d}\right)$, the bounded analytic functions on the polydisk [9]. To state their result, let us first define the Gleason distance between two points by

$$
\rho(\zeta, \lambda):=\sup \left\{|f(\zeta)|:\|f\|_{H^{\infty}\left(\mathbb{D}^{d}\right)} \leq 1, f(\lambda)=0\right\} .
$$

Notice that the elementary theory of Blaschke products on the disk shows that condition (3) in Theorem 0.1 is equivalent to:

(3)': There exists $c>0$ such that

$$
\prod_{j \neq i} \rho\left(\lambda_{i}, \lambda_{j}\right) \geq c
$$

for all $i$.

With respect to Lebesgue measure $\sigma$ on the distinguished boundary of the polydisk, one can define a space $H^{2}(\sigma)$ as the closure of the polynomials in $L^{2}(\sigma)$. This space has a reproducing kernel

$$
k(\lambda, z)=\prod_{n=1}^{d} \frac{1}{1-\bar{\lambda}^{n} z^{n}},
$$

where we use $\lambda^{n}$ to denote the $n^{\text {th }}$ component of the point $\lambda$.

Theorem 0.2 [Berndtsson, Chang and Lin] Consider the three statements

(1) There exists $c>0$ such that

$$
\prod_{j \neq i} \rho\left(\lambda_{i}, \lambda_{j}\right) \geq c
$$


for all $i$.

(2) The sequence $\left\{\lambda_{i}\right\}_{i=1}^{\infty}$ is an interpolating sequence for $H^{\infty}\left(\mathbb{D}^{d}\right)$.

(3) The sequence $\left\{\lambda_{i}\right\}_{i=1}^{\infty}$ is weakly separated and the associated Grammian with respect to Lebesgue measure $\sigma$ is bounded.

Then (1) implies (2) and (2) implies (3). Moreover the converse of both these implications is false.

For additional sufficient conditions that guarantee that a sequence is interpolating, see the papers of E. Kronstadt [14] and L. Huang [13].

It is the purpose of this paper to give a characterization of interpolating sequences for $H^{\infty}\left(\mathbb{D}^{2}\right)$. To this end, we observe that whereas all pure cyclic isometries are unitarily equivalent, so that in one variable one need only consider the Szegö kernel, there are many non-equivalent pairs of pure cyclic isometries. Thus when considering $H^{\infty}\left(\mathbb{D}^{2}\right)$ it is essential to consider a whole family of kernels simultaneously.

Given a kernel $k$ on the bidisk, say it is admissible if

$$
\left(1-\overline{\lambda^{1}} z^{1}\right) k(\lambda, z) \geq 0
$$

and

$$
\left(1-\overline{\lambda^{2}} z^{2}\right) k(\lambda, z) \geq 0
$$

i.e. if multiplication by each coordinate function is a contraction (see Section 1 below).

For a given sequence $\left\{\lambda_{i}\right\}_{i=1}^{\infty}$, the normalized Grammian of $k, G^{k}$, is then the infinite matrix

$$
G_{i j}^{k}=\frac{k\left(\lambda_{i}, \lambda_{j}\right)}{\sqrt{k\left(\lambda_{i}, \lambda_{i}\right) k\left(\lambda_{j}, \lambda_{j}\right)}} .
$$

Let $I$ denote the identity matrix $\delta_{i j}$, and $J$ the matrix all of whose entries are 1 . We can now state our main result.

Theorem 0.3 Let $\left\{\lambda_{i}\right\}_{i=1}^{\infty}$ be a sequence in $\mathbb{D}^{2}$. The following are equivalent:

(i) $\left\{\lambda_{i}\right\}_{i=1}^{\infty}$ is an interpolating sequence for $H^{\infty}\left(\mathbb{D}^{2}\right)$.

(ii) The following two conditions hold.

(a) For all admissible kernels $k$, their normalized Grammians are uniformly bounded:

$$
G^{k} \leq M I
$$

for some $M$. 
(b) For all admissible kernels $k$, their normalized Grammians are uniformly bounded below:

$$
N G^{k} \geq I
$$

for some $N$.

(iii) The sequence $\left\{\lambda_{i}\right\}_{i=1}^{\infty}$ is strongly separated and condition (a) alone holds.

(iv) Condition (b) alone holds.

Moreover, Condition (a) is equivalent to

$\left(a^{\prime}\right)$ : There exists a constant $M$ and positive semi-definite infinite matrices $\Gamma$ and $\Delta$ such that

$$
M \delta_{i j}-1=\Gamma_{i j}\left(1-\bar{\lambda}_{i}^{1} \lambda_{j}^{1}\right)+\Delta_{i j}\left(1-\bar{\lambda}_{i}^{2} \lambda_{j}^{2}\right) .
$$

Condition (b) is equivalent to

$\left(b^{\prime}\right)$ : There exists a constant $N$ and positive semi-definite infinite matrices $\Gamma^{\prime}$ and $\Delta^{\prime}$ such that

$$
N-\delta_{i j}=\Gamma_{i j}^{\prime}\left(1-\bar{\lambda}_{i}^{1} \lambda_{j}^{1}\right)+\Delta_{i j}^{\prime}\left(1-\bar{\lambda}_{i}^{2} \lambda_{j}^{2}\right)
$$

We prove Theorem 0.3 in Sections 2 and 3.

Compare Theorem 0.3 with the recent characterization, by D. Marshall and C. Sundberg and independently by C. Bishop, of the interpolating sequences for the multiplier algebra of the Dirichlet space, the space of analytic functions on $\mathbb{D}$ with finite Dirichlet integral. The reproducing kernel for this space is the Dirichlet kernel, $-\frac{1}{\bar{\lambda} z} \log (1-\bar{\lambda} z)$.

\section{Theorem 0.6 [Marshall-Sundberg, Bishop]}

A sequence $\left\{\lambda_{i}\right\}_{i=1}^{\infty}$ in $\mathbb{D}$ is an interpolating sequence for the multiplier algebra of the Dirichlet space if and only if it is weakly separated and the normalized Grammian of the Dirichlet kernel is bounded. Moreover, there are strongly separated sequences that are not interpolating sequences.

Possible strengthenings of Theorem 0.3 remain open, for example:

Question 0.7 If $\left\{\lambda_{i}\right\}_{i=1}^{\infty}$ is strongly separated, must it be an interpolating sequence for $H^{\infty}\left(\mathbb{D}^{2}\right)$ ? 


\section{Background on kernels}

By a kernel $k$ on a set $X$, we mean a function $k: X \times X \rightarrow \mathbb{C}$ that is positive definite, in the sense that

$$
\sum_{i, j=1}^{N} \overline{c_{i}} c_{j} k\left(\lambda_{i}, \lambda_{j}\right)>0
$$

for all $\lambda_{1}, \ldots, \lambda_{N}$ in $X$ and all complex numbers $c_{1}, \ldots, c_{N}$, unless $c_{1}=c_{2}=\ldots=c_{N}=0$.

We shall use $k_{\lambda}$ to denote the function $k(\lambda, \cdot)$, and call this function the kernel function at $\lambda$.

On the vector space of finite linear combinations of kernel functions, i.e. sums of the form $\sum c_{i} k_{\lambda_{i}}$, one can define an inner product by defining

$$
\left\langle k_{\lambda}, k_{z}\right\rangle=k(\lambda, z)
$$

and extending by sesqui-linearity. Completing this vector space with this inner product gives a Hilbert space of functions on $X$, which we shall denote by $\mathcal{H}_{k}$. For details of this construction, see [6]. Note that for any function $f$ in $\mathcal{H}_{k}$ the construction yields the reproducing property of the kernel:

$$
\left\langle f, k_{\lambda}\right\rangle=f(\lambda)
$$

The multiplier algebra of $\mathcal{H}_{k}$, denoted $M\left(\mathcal{H}_{k}\right)$, is the set of functions $\phi$ on $X$ with the property that whenever $f$ is in $\mathcal{H}_{k}$, then so is $\phi f$. It follows from the closed graph theorem that if $\phi$ is a multiplier of $\mathcal{H}_{k}$, then multiplication by $\phi$ is a bounded operator on $\mathcal{H}_{k}$, and the operator norm makes $M\left(\mathcal{H}_{k}\right)$ into a Banach algebra. We shall always consider $M\left(\mathcal{H}_{k}\right)$ with this norm.

The most well-known non-trivial example is the Szegö kernel. The Hilbert space produced is the Hardy space $H^{2}$, and its multiplier algebra is (isometrically) $H^{\infty}(\mathbb{D})$.

If $\phi$ is in $M\left(\mathcal{H}_{k}\right)$, let $M_{\phi}$ denote the operaor on $\mathcal{H}_{k}$ of multiplication by $\phi$. Notice that all the kernel functions are eigenvectors for the adjoint:

$$
M_{\phi}^{*} k_{\lambda}=\overline{\phi(\lambda)} k_{\lambda}
$$

as is seen by taking the inner product of either side with an arbitrary function in $\mathcal{H}_{k}$.

Notice too:

$$
\begin{array}{ccc} 
& \left\|M_{\phi}\right\| & \\
\Leftrightarrow & I-M_{\phi} M_{\phi}^{*} & \geq 0 \\
\Leftrightarrow & (1-\phi(z) \overline{\phi(\lambda)}) k(\lambda, z) & \geq 0 .
\end{array}
$$


Let us show that the boundedness of the Gram matrix is equivalent to a Carleson measure condition. The result is well-known.

Let $k_{i}$ denote the kernel function at $\lambda_{i}$, and $g_{i}$ denote the normalized kernel function at $\lambda_{i}$, so

$$
g_{i}=\frac{1}{\left\|k_{\lambda_{i}}\right\|} k_{\lambda_{i}}=\frac{k_{i}}{\left\|k_{i}\right\|}
$$

Proposition 1.1 Let $\left\{\lambda_{i}\right\}_{i=1}^{\infty}$ be a sequence in $X$. Then the following conditions are equivalent.

(BG) The associated Gram matrix has norm at most $C$.

(CM) The measure $\sum\left\|k_{i}\right\|^{-2} \delta_{\lambda_{i}}$ is a Carleson measure for $\mathcal{H}_{k}$, i.e. the following inequality holds:

$$
\sum_{i=1}^{\infty} \frac{\left|f\left(\lambda_{i}\right)\right|^{2}}{\left\|k_{i}\right\|^{2}} \leq C^{2}\|f\|_{\mathcal{H}_{k}}^{2} .
$$

ProOF: $(\mathrm{CM}) \Rightarrow(\mathrm{BG})$ :

$$
\begin{aligned}
\left\|\sum a_{i} g_{i}\right\|_{\mathcal{H}_{k}} & =\sup _{\|f\|=1}\left\langle\sum a_{i} g_{i}, f\right\rangle \\
& =\sup _{\|f\|=1} \sum a_{i}\left\|k_{i}\right\|^{-1} \overline{f\left(\lambda_{i}\right)} \\
& \leq \sup _{\|f\|=1}\left(\sum\left|a_{i}\right|^{2}\right)^{1 / 2}\left(\sum\left\|k_{i}\right\|^{-2}\left|f\left(\lambda_{i}\right)\right|^{2}\right)^{1 / 2} \\
& \leq C\left(\sum\left|a_{i}\right|^{2}\right)^{1 / 2} .
\end{aligned}
$$

$(\mathrm{BG}) \Rightarrow(\mathrm{CM})$ : Let $f$ be an arbitrary function in $\mathcal{H}_{k}$, and let $a_{i}=\left\|k_{i}\right\|^{-1} f\left(\lambda_{i}\right)$. Then

$$
\begin{aligned}
\sum\left\|k_{i}\right\|^{-2}\left|f\left(\lambda_{i}\right)\right|^{2} & =\left\langle f, \sum a_{i} g_{i}\right\rangle \\
& \leq\|f\|\left\|\sum a_{i} g_{i}\right\| \\
& \leq C\|f\|\left(\sum\left|a_{i}\right|^{2}\right)^{1 / 2} .
\end{aligned}
$$

As $\sum\left|a_{i}\right|^{2}=\sum\left\|k_{i}\right\|^{-2}\left|f\left(\lambda_{i}\right)\right|^{2}$, we get the desired inequality.

\section{Proofs of $(i) \Leftrightarrow(i i),(a) \Leftrightarrow\left(a^{\prime}\right)$ and $(b) \Leftrightarrow\left(b^{\prime}\right)$}

First, let us prove that (i) and (ii) are equivalent in Theorem 0.3.

Given an interpolating sequence $\left\{\lambda_{i}\right\}$, we shall call its interpolation constant the infimum of those numbers $M$ such that, whenever $\left|w_{i}\right| \leq 1$, there is a function $f$ of norm less than or equal to $M$ that interpolates each $\lambda_{i}$ to $w_{i}$. 
Lemma 2.1 The sequence $\left\{\lambda_{i}\right\}_{i=1}^{\infty}$ is an interpolating sequence for $H^{\infty}\left(\mathbb{D}^{2}\right)$ with interpolation constant $M$ if and only if, whenever $w_{i}$ is a sequence of complex numbers with $\sup \left|w_{i}\right| \leq 1$ and $k$ is an admissible kernel, then

$$
M^{2}\left\|\sum a_{i} k_{i}\right\|^{2} \geq\left\|\sum a_{i} w_{i} k_{i}\right\|^{2}
$$

whenever $\sum a_{i} k_{i}$ is in $\mathcal{H}_{k}$.

Proof: $(\Rightarrow)$. By hypothesis, there is a function $\phi$ of norm less than or equal to 1 in $H^{\infty}\left(\mathbb{D}^{2}\right)$ that maps $\lambda_{i}$ to $\bar{w}_{i} / M$. As $M_{z^{1}}$ and $M_{z^{2}}$ are commuting contractions on $\mathcal{H}_{k}$, by Andô's inequality [4], $\phi\left(M_{z^{1}}, M_{z^{2}}\right)=M_{\phi}$ is a contraction on $\mathcal{H}_{k}$. Therefore

$$
\begin{aligned}
0 & \leq M^{2}\left\langle\left(I-M_{\phi} M_{\phi}^{*}\right) k_{\lambda_{j}}, k_{\lambda_{i}}\right\rangle \\
& =M^{2}\left\langle k_{j}, k_{i}\right\rangle-w_{j} \bar{w}_{i}\left\langle k_{j}, k_{i}\right\rangle .
\end{aligned}
$$

But this means precisely that for any finite set of numbers $\left\{a_{i}\right\}$, we have

$$
M^{2}\left\langle\sum a_{j} k_{j}, \sum a_{i} k_{i}\right\rangle \geq\left\langle\sum a_{j} w_{j} k_{j}, \sum a_{i} w_{i} k_{i}\right\rangle
$$

and so (2.2) holds.

$(\Leftarrow)$ Conversely, a necessary and sufficient condition to be able to find a function $\phi$ in $H^{\infty}\left(\mathbb{D}^{2}\right)$ of norm at most $M$ that interpolates the points $\lambda_{i}$ to $\bar{w}_{i}$ is that, for every admissible kernel $k$,

$$
\left(M^{2}-w_{j} \bar{w}_{i}\right)\left\langle k_{j}, k_{i}\right\rangle \geq 0 .
$$

This was proved by the first author in [1]; see also [11, 7, 3]. So if $(2.2)$ holds for every admissible kernel and every choice of $w_{i}$, the sequence $\left\{\lambda_{i}\right\}$ is interpolating as desired.

Now, letting $w_{j}=\exp \left(2 \pi i t_{j}\right)$ and $a_{j}=c_{j}$ in (2.2) and integrating with respect to $t_{1}, t_{2}, \ldots$ on $[0,1] \times[0,1] \times \ldots$ one gets

$$
\sum_{j}\left|c_{j}\right|^{2}\left\|k_{j}\right\|^{2} \leq M^{2}\left\|\sum_{j} c_{j} k_{j}\right\|^{2}
$$

Similarly, letting $a_{j}=\exp \left(-2 \pi i t_{j}\right) c_{j}$ and $w_{j}=\exp \left(2 \pi i t_{j}\right)$ and integrating gives

$$
\left\|\sum_{j} c_{j} k_{j}\right\|^{2} \leq M^{2} \sum_{j}\left|c_{j}\right|^{2}\left\|k_{j}\right\|^{2}
$$

Combining (2.3) and (2.4), one gets that if $\left\{\lambda_{i}\right\}$ is an interpolating sequence, then for any normalized admissible kernel we have

$$
\frac{1}{M^{2}} \sum_{i}\left|c_{i}\right|^{2} \leq\left\|\sum_{i} c_{i} g_{i}\right\|^{2} \leq M^{2} \sum_{i}\left|c_{i}\right|^{2}
$$


(in other words, $\left\{g_{i}\right\}$ is a Riesz system, and the constant $M$ can be chosen uniformly). Conversely, if (2.5) holds, then (2.2) holds, with the constant $M^{2}$ replaced by $M^{4}$. As the first inequality in (2.5) says $G$ is bounded below by $1 / M$, and the second inequality says $G$ is bounded by $M$, we have shown:

The sequence $\left\{\lambda_{i}\right\}_{i=1}^{\infty}$ is an interpolating sequence with interpolation constant $M$.

$\Rightarrow \frac{1}{M} I \leq G^{k} \leq M I$ for all admissible kernels $k$.

$\Rightarrow$ The sequence $\left\{\lambda_{i}\right\}_{i=1}^{\infty}$ is an interpolating sequence with interpolation constant $M^{2}$.

To show that $\left(a^{\prime}\right) \Rightarrow(a)$ and $\left(b^{\prime}\right) \Rightarrow(b)$ is easy: take the Schur product (the entrywise product) of both sides of (0.4) with $G^{k}$, and one gets:

$$
M I-G^{k}=\Gamma \cdot\left[\left(1-\bar{\lambda}_{i}^{1} \lambda_{j}^{1}\right) \cdot G^{k}\right]+\Delta \cdot\left[\left(1-\bar{\lambda}_{i}^{2} \lambda_{j}^{2}\right) \cdot G^{k}\right]
$$

As the two quantities in brackets are positive, by definition of an admissible kernel, and the Schur product of two positive matrices is positive, one gets $G^{k} \leq M I$. Similarly, (0.5) gives $N G^{k} \geq I$.

The converse direction is a duality argument. Suppose $(a)$ holds. This can be expressed as saying:

Whenever $k(\lambda, z)$ is a kernel such that:

$$
\text { and } \begin{aligned}
&\left(1-\overline{\lambda^{1}} z^{1}\right) \cdot k \geq 0 \\
&\left(1-\overline{\lambda^{2}} z^{2}\right) \cdot k \geq 0 \\
& \text { then } \\
&(M I-J) \cdot k \geq 0
\end{aligned}
$$

where we use - to denote the Schur product. Now a Hahn-Banach argument shows that $M I-J$ cannot be separated from the closed wedge of infinite matrices of the form

$$
\Gamma(\lambda, z) \cdot\left(1-\bar{\lambda}^{1} z^{1}\right)+\Delta(\lambda, z) \cdot\left(1-\bar{\lambda}^{2} z^{2}\right) \quad: \quad \Gamma \geq 0, \Delta \geq 0 .
$$

Indeed, fix a positive integer $n$. Let $\mathcal{T}_{n}$ be the set of all $n$-by- $n$ self-adjoint matrices $T$ representable in the form

$$
T_{i j}=\left(1-\lambda_{i}^{1} \overline{\lambda_{j}^{1}}\right) \Gamma_{i j}+\left(1-\lambda_{i}^{2} \overline{\lambda_{j}^{2}}\right) \Delta_{i j}
$$

where $\Gamma$ and $\Delta$ are positive. As $\mathcal{T}_{n}$ is a closed wedge, if $M I-J$ were not in $\mathcal{T}_{n}$, there would be a real linear functional on the space of all $n$-by- $n$ self-adjoint matrices that was positive on $\mathcal{T}_{n}$ and strictly negative on $M I-J$. 
Any such linear functional must be of the form $T \mapsto \operatorname{tr}(W T)$ for some self-adjoint matrix $W$. As $\mathcal{T}_{n}$ contains the set of all positive matrices (let $\Delta=0$ and $\Gamma$ be the Schur product of the positive matrix $1 /\left(1-\overline{\lambda_{i}^{1}} \lambda_{j}^{1}\right)$ with an arbitrary positive matrix), $W$ must be positive. Let $K$ be the transpose of $W$. Then

$$
\sum_{i, j=1}^{n} c_{i} \overline{c_{j}} K_{i j}\left(1-\lambda_{i}^{1} \overline{\lambda_{j}^{1}}\right)=\operatorname{tr}(W T)
$$

where

$$
T_{i j}=\left(1-\lambda_{i}^{1} \overline{\lambda_{j}^{1}}\right) c_{i} \overline{c_{j}}
$$

is in $\mathcal{T}_{n}$. So $K$ satisfies (2.6), and similarly also (2.7). Therefore $(M I-J) \cdot K$ is positive, so

$$
\operatorname{tr}(W(M I-J))=\sum_{i, j=1}^{n} K\left(\lambda_{i}, \lambda_{j}\right)\left(M \delta_{i j}-1\right) \geq 0
$$

a contradiction if $M I-J$ is not in $\mathcal{T}_{n}$. So for every $n$, we have $M I-J$ is in $\mathcal{T}_{n}$; it follows e.g. from Kurosh's theorem [5, p.75] that there is a choice of $\Gamma$ and $\Delta$ such that

$$
M \delta_{i j}-1=\Gamma_{i j}\left(1-\bar{\lambda}_{i}^{1} \lambda_{j}^{1}\right)+\Delta_{i j}\left(1-\bar{\lambda}_{i}^{2} \lambda_{j}^{2}\right)
$$

for all $i, j$.

A similar argument shows that $(b) \Rightarrow\left(b^{\prime}\right)$.

\section{Proof that $(i i i) \Leftrightarrow(i v)$.}

Let us analyze condition $\left(b^{\prime}\right)$. First some notation. Given Hilbert spaces $\mathcal{L}_{1}$ and $\mathcal{L}_{2}$, we let $B\left(\mathcal{L}_{1}, \mathcal{L}_{2}\right)$ denote the bounded linear operators from $\mathcal{L}_{1}$ to $\mathcal{L}_{2}$, and $H^{\infty}\left(\mathbb{D}^{2}, B\left(\mathcal{L}_{1}, \mathcal{L}_{2}\right)\right)$ the space of bounded holomorphic functions from $\mathbb{D}^{2}$ to $B\left(\mathcal{L}_{1}, \mathcal{L}_{2}\right)$. Let $\left\{e_{i}\right\}_{i=1}^{\infty}$ be the usual orthonormal basis of $l^{2}$, the vector with 1 in the $i^{\text {th }}$ slot and 0 elsewhere.

Lemma 3.1 With notation as in Theorem 0.3, condition ( $\left.b^{\prime}\right)$ is equivalent to:

$(b ")$ : There exists a function $\Phi$ in $H^{\infty}\left(\mathbb{D}^{2}, B\left(\mathbb{C}, l^{2}\right)\right)$ of norm at most $\sqrt{N}$ such that $\Phi\left(\lambda_{i}\right)=e_{i}$.

Before proving this lemma, we need to recall the following theorem of the first author $[2]:$ 
Theorem 3.2 The function $\Psi$ is in the closed unit ball of $H^{\infty}\left(\mathbb{D}^{2}, B\left(\mathcal{L}_{1}, \mathcal{L}_{2}\right)\right)$ if and only if: there are auxiliary Hilbert spaces $\mathcal{H}_{1}$ and $\mathcal{H}_{2}$ and an isometry $U: \mathcal{L}_{1} \oplus \mathcal{H}_{1} \oplus \mathcal{H}_{2}: \rightarrow$ $\mathcal{L}_{2} \oplus \mathcal{H}_{1} \oplus \mathcal{H}_{2}$ such that, with respect to the decomposition of $U$ as

$$
\begin{aligned}
& \mathcal{L}_{1} \quad \mathcal{H}_{1} \oplus \mathcal{H}_{2} \\
& U=\begin{array}{l}
\mathcal{L}_{2} \\
\mathcal{H}_{1} \oplus \mathcal{H}_{2}
\end{array}\left(\begin{array}{ll}
A & B \\
C & D
\end{array}\right)
\end{aligned}
$$

we have

$$
\Psi(\lambda)=A+B E_{\lambda}\left(1-D E_{\lambda}\right)^{-1} C .
$$

Here, for $\lambda=\left(\lambda^{1}, \lambda^{2}\right)$ in $\mathbb{D}^{2}, E_{\lambda}=\lambda^{1} I_{\mathcal{H}_{1}} \oplus \lambda^{2} I_{\mathcal{H}_{2}}$ is the operator of multiplication by $\lambda^{1}$ on $\mathcal{H}_{1}$ and multiplication by $\lambda^{2}$ on $\mathcal{H}_{2}$.

Proof of Lemma 3.1: Consider condition $\left(b^{\prime}\right)$ :

$$
N-\delta_{i j}=\Gamma_{i j}^{\prime}\left(1-\bar{\lambda}_{i}^{1} \lambda_{j}^{1}\right)+\Delta_{i j}^{\prime}\left(1-\bar{\lambda}_{i}^{2} \lambda_{j}^{2}\right)
$$

Choose vectors $f_{i}$ and $g_{i}$ in auxiliary Hilbert spaces $\mathcal{H}_{1}$ and $\mathcal{H}_{2}$ so that

$$
\begin{aligned}
& \left\langle f_{j}, f_{i}\right\rangle=\Gamma_{i j}^{\prime} \\
& \left\langle g_{j}, g_{i}\right\rangle=\Delta_{i j}^{\prime} .
\end{aligned}
$$

Then Equation 3.3 can be rewritten as

$$
N+\bar{\lambda}_{i}^{1} \lambda_{j}^{1}\left\langle f_{j}, f_{i}\right\rangle+\bar{\lambda}_{i}^{2} \lambda_{j}^{2}\left\langle g_{j}, g_{i}\right\rangle=\left\langle e_{j}, e_{i}\right\rangle+\left\langle f_{j}, f_{i}\right\rangle+\left\langle g_{j}, g_{i}\right\rangle .
$$

Letting $h_{i}=f_{i} \oplus g_{i}$, Equation 3.4 becomes

$$
\left\langle\left(\begin{array}{c}
\sqrt{N} \\
E_{\lambda_{j}} h_{j}
\end{array}\right),\left(\begin{array}{c}
\sqrt{N} \\
E_{\lambda_{i}} h_{i}
\end{array}\right)\right\rangle=\left\langle\left(\begin{array}{c}
e_{j} \\
h_{j}
\end{array}\right),\left(\begin{array}{c}
e_{i} \\
h_{i}
\end{array}\right)\right\rangle .
$$

So there is an isometry

$$
L:\left(\begin{array}{c}
\sqrt{N} \\
E_{\lambda_{i}} h_{i}
\end{array}\right) \mapsto\left(\begin{array}{c}
e_{i} \\
h_{i}
\end{array}\right) .
$$

Increasing $\mathcal{H}_{1}$ and $\mathcal{H}_{2}$ if necessary, $L$ can be extended to a unitary $U: \mathbb{C} \oplus \mathcal{H}_{1} \oplus \mathcal{H}_{2} \rightarrow$ $l^{2} \oplus \mathcal{H}_{1} \oplus \mathcal{H}_{2}$. Write

$$
\left.U=l_{\mathcal{H}_{1} \oplus \mathcal{H}_{2}} \begin{array}{cc}
\mathbb{C} & \mathcal{H}_{1} \oplus \mathcal{H}_{2} \\
A & B \\
C & D
\end{array}\right),
$$


and let $\Psi(\lambda)=A+B E_{\lambda}\left(1-D E_{\lambda}\right)^{-1} C$. Then, by Theorem 3.2, $\|\Psi\| \leq 1$. Solving

$$
\left(\begin{array}{cc}
A & B \\
C & D
\end{array}\right)\left(\begin{array}{c}
\sqrt{N} \\
E_{\lambda_{i}} h_{i}
\end{array}\right)=\left(\begin{array}{c}
e_{i} \\
h_{i}
\end{array}\right),
$$

we get $\Psi\left(\lambda_{i}\right)=\frac{1}{\sqrt{N}} e_{i}$. Then $\Phi=\sqrt{M} \Psi$ is the required function.

Conversely, if $\left(b^{\prime \prime}\right)$ holds, let $\Psi=\frac{1}{\sqrt{N}} \Phi$, and write $\Psi$ as in Theorem 3.2. Then Equation 3.6 holds, and hence going backwards so do Equations 3.5, 3.4 and 3.3.

A similar argument shows:

Lemma 3.7 With notation as in Theorem 0.3, condition ( $\left.a^{\prime}\right)$ is equivalent to:

$(a ")$ : There exists a function $\Psi$ in $H^{\infty}\left(\mathbb{D}^{2}, B\left(l^{2}, \mathbb{C}\right)\right)$ of norm at most $\sqrt{M}$ such that $\Psi\left(\lambda_{i}\right) e_{i}=1$.

Now, suppose condition $\left(b^{\prime \prime}\right)$ holds. Letting $\Psi(\lambda)=\Phi(\lambda)^{t}$, we get $\left(a^{\prime \prime}\right)$. Moreover, writing $\Phi$ as

$$
\Phi(\lambda)=\left(\begin{array}{c}
\phi_{1} \\
\phi_{2} \\
\vdots
\end{array}\right),
$$

we get a sequence of functions $\phi_{i}$ such that $\sum_{i}\left|\phi_{i}(\lambda)\right|^{2} \leq N$ and $\phi_{i}\left(\lambda_{j}\right)=\delta_{i j}$. So in particular, $\left\{\lambda_{i}\right\}$ is strongly separated, proving that $(i v) \Rightarrow(i i i)$.

Conversely, suppose $(i i i)$ holds. By $\left(a^{\prime \prime}\right)$, writing

$$
\Psi=\left(\psi_{1}, \psi_{2}, \ldots\right),
$$

we have $\sum_{i}\left|\psi_{i}(\lambda)\right|^{2} \leq M$ and $\psi_{i}\left(\lambda_{i}\right)=1$. Moreover, strong separation means we have a sequence of functions $\chi_{i}$ such that $\chi_{i}\left(\lambda_{j}\right)=\delta_{i j}$ and $\left\|\chi_{i}\right\| \leq C$ for all $i$. Letting $\phi_{i}=\psi_{i} \chi_{i}$, and

$$
\Phi(\lambda)=\left(\begin{array}{c}
\phi_{1} \\
\phi_{2} \\
\vdots
\end{array}\right),
$$

we get $\|\Phi\| \leq C \sqrt{M}$, and $\Phi\left(\lambda_{i}\right)=e_{i}$. So $\left(b^{\prime \prime}\right)$ holds with constant $C \sqrt{M}$, proving $(i i i) \Rightarrow$ (iv).

Remark: A theorem due to Varopoulos [15] and Bernard [8] (see also [12, p. 298]), which applies to any uniform algebra that is also a dual space, asserts that given an interpolating sequence $\left\{\lambda_{i}\right\}_{i=1}^{\infty}$, one can find functions $\phi_{i}$ with $\phi_{i}\left(\lambda_{j}\right)=\delta_{i j}$ and $\sum\left|\phi_{i}(z)\right| \leq M^{2}$ for all $z$. These are sometimes called Per Beurling functions, because he showed they existed for all interpolating sequence on the disk (see [12, p. 294]). The equivalence of Condition ( $\left.b^{\prime \prime}\right)$ and a sequence being interpolating is then the $H^{\infty}\left(\mathbb{D}^{2}\right)$ case of the Varopoulos-Bernard theorem. 


\section{References}

[1] J. Agler. Some interpolation theorems of Nevanlinna-Pick type. Preprint, 1988.

[2] J. Agler. On the representation of certain holomorphic functions defined on a polydisc. In Operator Theory: Advances and Applications, Vol. 48, pages 47-66. Birkhäuser, Basel, 1990.

[3] J. Agler and J.E. McCarthy. Nevanlinna-Pick interpolation on the bidisk. J. Reine Angew. Math., 506:191-204, 1999.

[4] T. Andô. On a pair of commutative contractions. Acta Sci. Math. (Szeged), 24:88-90, 1963.

[5] A.V. Arkhangel'skii and L.S. Pontryagin (eds.). General Topolgy I. Springer, Berlin, 1990.

[6] N. Aronszajn. Theory of reproducing kernels. Trans. Amer. Math. Soc., 68:337-404, 1950.

[7] J.A. Ball and T.T. Trent. Unitary colligations, reproducing kernel Hilbert spaces, and Nevanlinna-Pick interpolation in several variables. J. Funct. Anal., 197:1-61, 1998.

[8] A. Bernard. Algèbres quotients d'algèbres uniformes. C.R. Acad. Sci. Paris, Sér. A, 272:1101-1104, 1971.

[9] B. Berndtsson, S.-Y. Chang, and K.-C. Lin. Interpolating sequences in the polydisk. Trans. Amer. Math. Soc., 302:161-169, 1987.

[10] L. Carleson. An interpolation problem for bounded analytic functions. Amer. J. Math., 80:921-930, 1958.

[11] B.J. Cole and J. Wermer. Pick interpolation, von Neumann inequalities, and hyperconvex sets. In Complex Potential Theory, pages 89-129. Kluwer Acad. Publ., Dordrecht, 1994.

[12] John B. Garnett. Bounded Analytic Functions. Academic Press, New York, 1981.

[13] L.F. Huang. $H^{\infty}$ interpolating sequences in polydisks. Compl. Var. Theory Appl., 18:243-251, 1992. 
[14] E.P. Kronstadt. Interpolating sequences in polydisks. Trans. Amer. Math. Soc., 199:369-398, 1974.

[15] N.Th. Varopoulos. Ensembles pics et ensembles d'interpolation pour les algèbres uniformes. C.R. Acad. Sci. Paris, Sér. A, 272:866-867, 1971. 